\title{
Hüftfraktur - und wie geht es weiter?
}

\author{
Im Alter steigt das Frakturrisiko bei Patienten mit Osteoporose rasant an. Wirksame \\ Primär- und Sekundärprophylaxe sind gefragt. Wie diese leitliniengerecht erfolgen \\ sollten, erläutert Prof. H. J. Heppner in diesem Beitrag.
}

? Eine 77-jährige Patientin stellt sich nach der Entlassung aus der Klinik, wo eine pertrochantäre Oberschenkelfraktur operativ versorgt worden ist, in der Sprechstunde vor. Nun stellt sich die Frage nach einer Osteoporosetherapie. Wie soll ich weiter vorgehen? Ist die Knochendichtemessung unabdingbar?

I Diagnostik und Therapie der Osteoporose ist beim geriatrischen Patienten von großer Bedeutung. Hierzu zählt nicht nur die medikamentöse Behandlung, sondern auch die gezielte Bewegungstherapie und Sturzprävention. Ziel ist es, Hüft- oder Wirbelkörperfrakturen zu vermeiden. In der Realität ist die Fraktur nach einem Sturz leider meist die Erstmanifestation einer bereits seit Jahren bestehenden, nicht erkannten Osteoporose. Bei Frauen über 70 Jahren und Männern über 80 Jahren nimmt das Frakturrisiko bei Osteoporose rasant zu.

Die Leitlinie des Dachverbandes Osteologie (DVO) empfiehlt die Bisphos- phonate Alendronat, Risedronat und Zoledronat ebenso wie Denosumab (monoklonaler Antikörper mit antiresorptiver Wirkung durch gezielte Hemmung der Osteoklastenaktivität) als Erstlinientherapie. Für diese Medikamente konnte eine signifikante Reduktion des Frakturrisikos bei geriatrischen Patienten nachgewiesen werden.

Liegt die 25-Hydroxy-Vitamin-D-Serum-Konzentration unter $20 \mathrm{ng} / \mathrm{ml}$, ist von einem erhöhten Frakturrisiko auszugehen. Vitamin D hat zusätzlich Effekte auf Muskelkraft, Koordination und Sturzverhütung und senkt damit das Frakturrisiko. Gleiches gilt für Kalzium. Somit sind die Gabe von Vitamin D 800 IE täglich und Kalzium 500-1.000 mg täglich sowie einmal jährlich ein Screening zum Sturzrisiko und ein geriatrisches Assessment bei allen über 70-Jährigen indiziert.

Calcitonin hat keinen Stellenwert in der First-line-Therapie der Osteoporose beim geriatrischen Patienten und Teri- paratid (Parathormon) ist ein Reservetherapeutikum.

Zwar geben die Leitlinien der DVO die Osteodensiometrie (DXA) als Standard vor, lassen aber optional den Trabecular Bone Score (TBS) zu. Außerdem gilt die Empfehlung, dass eine stattgehabte Hüftfraktur oder zwei typische Frakturen im Röntgenbild die Einleitung einer medikamentösen Osteoporosetherapie ohne vorherige Knochendichtemessung beim geriatrischen Patienten rechtfertigen.

\section{Zurück zum Fall}

Bei Ihrer Patientin sollten Sie eine Bisphosphonattherapie mit Kalzium- und Vitamin-D-Substitution einleiten. Zusätzlich ist ein Mobilitätsassessment zur Abschätzung des Sturzrisikos alle sechs Monate empfehlenswert.

$\rightarrow$ Anschrift des Verfassers: Univ.-Prof. Dr. med. H. J. Heppner, MHBA Klinik für Geriatrie HELIOS Klinikum Schwelm, Universität Witten/Herdecke Dr.-Moeller-Str. 15, D-58332 Schwelm E-Mail: hans.heppner@uni-wh.de

Tab. 1 Medikamente zur frakturreduzierenden Osteoporosetherapie

\begin{tabular}{|c|c|c|c|}
\hline Substanz & Dosis & Applikation & Besonderheiten \\
\hline Alendronat & $\begin{array}{l}70 \mathrm{mg} \\
1 \times \text { wöchentlich }\end{array}$ & oral & $\begin{array}{l}\text { spezielle Einnahmevorschriften, keine Einnahme } \\
\text { bei GFR }<35 \mathrm{ml} / \mathrm{min}\end{array}$ \\
\hline Risedronat & $\begin{array}{l}35 \mathrm{mg} \\
1 \times \text { wöchentlich }\end{array}$ & oral & $\begin{array}{l}\text { spezielle Einnahmevorschriften, keine Einnahme } \\
\text { bei GFR }<30 \mathrm{ml} / \mathrm{min}\end{array}$ \\
\hline $\begin{array}{l}\text { Strontiumra- } \\
\text { nelat }\end{array}$ & $2 \mathrm{~g}, 1 \times$ täglich & oral & $\begin{array}{l}\text { bei schwerer Osteoporose, wenn eine Therapie mit } \\
\text { anderen Substanzen nicht möglich ist }\end{array}$ \\
\hline Zoledronat & 5 mg, 1 x jährlich & intravenös & 2 Wochen Abstand zur Fraktur-Op. \\
\hline Ibandronat & $\begin{array}{l}3 \mathrm{mg} \\
\text { alle } 3 \text { Monate }\end{array}$ & intravenös & $\begin{array}{l}\text { Kalzium und Vitamin D müssen begleitend } \\
\text { verabreicht werden }\end{array}$ \\
\hline Denosumab & $\begin{array}{l}60 \mathrm{mg} \\
\text { alle } 6 \text { Monate }\end{array}$ & subcutan & $\begin{array}{l}\text { auch andere Dosierungen erhältlich, Kalzium und } \\
\text { Vitamin D müssen begleitend verabreicht werden }\end{array}$ \\
\hline Teriparatid & $20 \mu \mathrm{g}, 1 \times$ täglich & subcutan & second-line-Therapie, max. 24 Monate lang \\
\hline
\end{tabular}

Haben Sie auch eine Frage an das consilium Geriatrie?

Haben Sie auch eine Frage, speziell aus den Bereichen Kognitive Störungen, Neurologie, Infektiologie, Schlafstörungen oder Dysphagie im Alter? Prof. Heppner und sein Expertenteam beantworten diese gerne. Das "consilium Geriatrie" ist ein gemeinsamer Service der MMW und des Unternehmens InfectoPharm.

Schicken Sie Ihre Frage kostenfrei an: geriatrie@consilium.services 